\title{
Efficient Deadlock Detection in Parallel Computer Systems with Wormhole Routing
}

\author{
Soojung Lee \\ GyeongIn National University of Education \\ 6-8 Seoksu-dong, Anyang, Korea 430-739 \\ sjlee@gin.ac.kr
}

\begin{abstract}
Wormhole routing has been popular in massively parallel computing systems due to its low packet latency. However, it is subject to deadlock, where packets are waiting for resources in a cyclic form indefinitely. Current deadlock detection techniques are basically dependent on the time-out strategy, thus yielding unignorable number of false deadlock detections especially in heavy network loads or with long packets. Moreover, several packets in a deadlock may be marked as deadlocked, which would saturate the resources allocated for recovery. This paper proposes a simple but more accurate deadlock detection scheme which is less dependent on the time-out value. The proposed scheme presumes deadlock only when a cyclic dependency among blocked packets exists. Consequently, the suggested scheme considerably reduces the probability of detecting false deadlocks over previous schemes, thus enabling more efficient deadlock recovery and higher network throughput. Simulation results are provided to demonstrate the efficiency of the proposed scheme.
\end{abstract}

\section{Introduction}

Wormhole routing has been quite popular in parallel computing systems with interconnection networks, because it can significantly reduce packet latency and the requirement of packet buffers is obviated [1. In wormhole routing, a packet is split into several flits for transmission. A header flit leads the route and the remaining flits follow in a pipelined fashion. However, it is susceptible to deadlock, where a set of packets may become blocked forever. This situation occurs when each packet in the set requests a channel resource held by another packet in the set in a circular way.

Deadlock avoidance has been a traditional approach in handling deadlock problem 2]. In this approach, routing is restricted in a way that no cyclic dependency exists between channels. For example, the turn model [36] prohibits turns that may form a cycle. However, such design of routing algorithm results in low adaptivity and increased latency. A routing algorithm is said to be adaptive if a routing path is selected based on dynamic network conditions.

A way to have higher throughput while avoiding deadlock is using the virtual channel. A number of virtual channels share a physical channel, thereby composing virtual networks and facilitating adaptive routing algorithms. In [4, virtual 
channels are divided into two classes; one for dimension-order routing with no cyclic dependency and the other for fully adaptive minimal routing. Although this scheme can provide more flexibility, it is only partially adaptive.

The frequency of deadlock occurrence is reported to be very low with a fully adaptive routing algorithm 11. Hence, it is wasteful to limit routing adaptivity for rarely occurring deadlocks. This motivated a new approach to handling deadlocks, deadlock detection and recovery. The criteria for determining deadlock is basically time-out. That is, a packet is presumed as deadlocked if it has been waiting for longer than a given threshold 7]10 or if all of its requested channels are inactive for longer than the threshold 9 . Although these schemes can detect all deadlocks, they may misinterpret simply-congested packets as deadlocked. A more sophisticated method to determine deadlock was proposed in 8 , which, to our knowledge, performs best in detecting deadlocks accurately. It notices a sequence of blocked packets as a tree whose root is a packet that is advancing. When the root becomes blocked later, only the packet blocked due to the root is eligible to recover. However, the accuracy of the mechanism in 8 relies on the dependency configuration of blocked packets as well as the threshold value. In general, deadlock is recovered by ejecting deadlocked packets from the network [8] or by forwarding them through a dedicated deadlock-free path [10.

Deadlock frequency determines the performance of deadlock detection and recovery schemes. In heavily loaded networks, those packets presumed as deadlocked will saturate the recovery resources, thus degrading performance considerably. Therefore, it is required that only real-deadlocked packets use the resources, as their occurrence frequency is low [11. However, previous schemes 78/9] cannot distinguish between real deadlocked and blocked packets waiting longer than the given threshold. Also, they force all the packets in deadlock to recover, although it is sufficient to choose only one packet to break the deadlock.

The performance of a fully adaptive routing algorithm relies on the effectiveness of the deadlock detection mechanism associated with it. We propose simple but effective deadlock detection mechanisms which employ a special control packet named probe to detect deadlock. A blocked packet initiates a probe when all of its requested channels are inactive for the threshold and propagates it along the path of inactive channels. The presence of deadlock is presumed, if a cyclic dependency among blocked packets is suspected through probe propagation, thereby reducing the number of packets detected as deadlocked considerably over previous schemes. The performance of our schemes is simulated and compared with that of a previous scheme [8], known to be most efficient in reducing the number of false deadlock detections.

\section{The Proposed Mechanism}

We first describe our scheme for mesh networks. To depict resource dependencies at a point of time, the channel wait-for graph (CWFG) can be used, where vertices represent the resources (either virtual channels or physical channels for networks with no virtual channel) and edges represent either 'wait-for' or 'owned-after' relations [511/12. A wait-for edge $\left(c_{i}, c_{j}\right)$ represents that there 
exists a message occupying channel $c_{i}$ and waiting for channel $c_{j}$. An owned-after edge $\left(c_{i}, c_{j}\right)$ implies the temporal order in which the channels were occupied, i.e., $c_{j}$ is owned after $c_{i}$. In a network with virtual channels, a packet header may proceed if any of the virtual channels associated with its requested channel is available. Hence, in such network, there are multiple wait-for edges outgoing from a vertex, while there always exists only one owned-after edge, as data flits in wormhole networks simply follow their previous flits in a pipelined fashion. For the description of the proposed mechanism, we introduce the following notation.

Notation 1. Assume that blocked packet $m$ holds $c$ and an edge $\left(c, c^{\prime}\right)$ exists in the CWFG. We refer to $c^{\prime}$ as a predecessor of $c$ with respect to $m$ and denote the set of predecessors of $c$ with respect to $m$ as $\left.\operatorname{pred}(c)\right|_{m}$. Also, let $\operatorname{dim}(c)$ and $\operatorname{dir}(c)$ denote the dimension and direction of a physical or virtual channel $c$, respectively.

As a cycle is a necessary condition to form a deadlock, our scheme is motivated by a simple observation that a cycle involves at least four blocked packets in a minimal routing. From this observation, one may think of an idea that a cycle is detected by counting the number of blocked packets in sequence. That is, if the number counts up to at least four, one concludes that a potential deadlock exists. This idea obviously reduces the number of false deadlock detections over those schemes which simply measure the channel inactivity time for time-out for deadlock detection; these schemes would yield deadlock detections as much as the number of blocked packets.

Obviously, our idea may detect deadlock falsely. For instance, consider a sequence of blocked packets residing within one dimension only, without turning to other dimensions; note that such sequence of blocked packets cannot form a cycle in meshes. Our idea would declare deadlock in such case, although there is none. Therefore, in order to further reduce the number of false deadlock detections, we take a different view of identifying a cycle. Namely, we focus on the number of corners, rather than on the number of blocked packets in sequence. If the number of corners formed by a sequence of blocked packets counts up to four or more, the presence of a deadlock is presumed.

The above criteria for detecting deadlock based on the number of turns are likely to detect deadlock falsely. However, the frequency of deadlock occurrence is reported to be very low with a fully adaptive routing algorithm 11 . Hence, it is believed that a complex cycle would rarely occur except in a heavy network condition. Moreover, it is more important to quickly dissipate congestion by resolving simple cycles before they develop into complex ones.

To implement the above idea, we employ a special control packet named probe to traverse along inactive channels for deadlock detection. Basically a probe is initiated upon time-out. However, in order not to initiate probes repetitively along the same channel, a bit, named PIB (Probe Initiation Bit), is allocated for each physical channel to indicate that a probe is initiated and transmitted through the channel. The bit is reset when the physical channel becomes active. Specifically, probes are initiated and propagated according to the following rules. 
Rule 1. A router initiates a probe if

(i) there is a blocked packet,

(ii) all the channels requested by the blocked packet are inactive for threshold TO due to other blocked packets holding the channels, and

(iii) PIB of any one of the channels requested by the blocked packet is zero.

Let $c$ be a channel with zero PIB, requested by the blocked packet. Also, let $m$ be one of the packets holding $c$. Upon initiation, the router transmits $\operatorname{probe}(m)$ through $c$ and sets the PIB of $c$ to one.

Rule 2. When a packet is delivered along a channel with PIB of one, set the PIB to zero.

Rule 3. Let $c$ be the input channel through which probe $(m)$ is received. Let $e$ be $\left(c, c^{\prime}\right)$, where $\left.c^{\prime} \in \operatorname{pred}(c)\right|_{m}$. If $e$ is an owned-after edge, simply forward probe $(m)$ through $c^{\prime}$. Otherwise if $e$ is a wait-for edge, check if all the channels requested by $m$ are inactive for threshold $T O_{F}$ due to other blocked packets holding the channels. If yes, transmit probe $\left(m^{\prime}\right)$ through $c^{\prime}$, where $m^{\prime}$ is one of the packets holding $c^{\prime}$. If no, discard the received probe $(m)$.

By Rule 3, a probe follows the path through which a blocked packet is routed until the header of the packet is met. At that moment, all the channels requested by the header are checked for their inactivity time. When the time exceeds $T O_{F}$ threshold for each of the channels, the probe is forwarded through one of the channels. Unlike $T O$, one may set $T O_{F}$ to a small value, in order not to delay the probe transmission.

Let us call the process of initiation and transmission of a probe probing. The probe carries the information on the number of turns made by blocked packets which hold the channels on the probing path; a packet is said to make turn if it changes its routing dimension. The number of turns is represented by count. When a router receives a probe, it examines count carried by the probe If count is at least four, the router presumes the presence of deadlock. As count is carried by probes, we name this mechanism COUNTING scheme. Specifically, the mechanism manipulates count as follows.

Rule C1. Upon initiation of a probe for blocked packet $m$ waiting on input channel $c$, if the probe is to be transmitted along channel $c^{\prime}$, then

(i) if $\operatorname{dim}(c)=\operatorname{dim}\left(c^{\prime}\right)$, then transmit the probe carrying count of zero along $c^{\prime}$.

(ii) otherwise transmit the probe carrying count of one along $c^{\prime}$.

Rule C2. Upon receiving a probe through channel $c$, if the probe is to be transmitted along channel $c^{\prime}$, then

(i) if $\operatorname{dim}(c) \neq \operatorname{dim}\left(c^{\prime}\right)$, then increase the received count by one.

(ii) if count $\geq 4$ and $\left(c, c^{\prime}\right)$ is a wait-for edge, then declare deadlock; otherwise, transmit the probe carrying count along $c^{\prime}$.

In Rules $\mathrm{C} 1$ and $\mathrm{C} 2$, whether to send the probe or not and which channel to send the probe through are all determined by Rules 1 and 3. Rule $\mathrm{C} 2$ allows deadlock 
declaration when the probe encounters a packet header. This is to recover the potential deadlock by selecting that packet as a victim.

Note that COUNTING scheme does not consider directions of turns. It may misinterpret a non-cyclic sequence of blocked packets involving turns of the same direction as deadlock. In order to better distinguish deadlock, we suggest a slight modification to COUNTING scheme that reflects the direction of turns. A bit is used for each direction in each dimension. For example, four bits are used for 2D networks; two bits for positive and negative directions in dimension zero and another two bits in dimension one. In general, $2 n$ bits are used for $n \mathrm{D}$ networks. These bits are carried by probes as count is carried in COUNTING scheme. The basic idea of the modified scheme is set the bits corresponding to the turn direction and declare deadlock when at least four bits corresponding to any two dimensions are set. We call this modified scheme BITSET scheme. The basic operations for probe and PIB management are the same as described in Rules 1 to 3 . Hence, we present only bit operations below.

Notation 2. Probes in BITSET scheme carry $2 n$ TBs (Turn Bits) in $n \mathrm{D}$ networks. Specifically, $T B_{d},+$ and $T B_{d}$, - represent bits for positive and negative directions in dimension $d$, respectively.

Rule B1. Upon initiation of a probe for blocked packet $m$ waiting on input channel $c$, if the probe is to be transmitted along channel $c^{\prime}$, then

(i) if $\operatorname{dim}(c)=\operatorname{dim}\left(c^{\prime}\right)$, then transmit the probe along $c^{\prime}$ carrying zero $T B \mathrm{~s}$.

(ii) otherwise transmit the probe along $c^{\prime}$ carrying $T B$ s with $T B_{\operatorname{dim}(c), \operatorname{dir}(c)}$ and $T B_{\operatorname{dim}\left(c^{\prime}\right), \operatorname{dir}\left(c^{\prime}\right)}$ set, where $\operatorname{dir}(c)=+$ if packet $m$ was sent along positive direction of $c$. Otherwise, $\operatorname{dir}(c)=-. \operatorname{dir}\left(c^{\prime}\right)$ is set similarly.

Rule B2. Upon receiving a probe through channel $c$, if the probe is to be transmitted along channel $c^{\prime}$, then

(i) if $\operatorname{dim}(c) \neq \operatorname{dim}\left(c^{\prime}\right)$, then set $T B_{\operatorname{dim}(c), \operatorname{dir}(c)}$ and $T B_{\operatorname{dim}\left(c^{\prime}\right), \operatorname{dir}\left(c^{\prime}\right)}$.

(ii) if $T B_{d 1,+}, T B_{d 1,-}, T B_{d 2,+}$, and $T B_{d 2,-}$, for any two dimensions $d 1$ and $d 2$, are set and $\left(c, c^{\prime}\right)$ is a wait-for edge, then declare deadlock; otherwise, transmit the probe carrying $T B$ s along $c^{\prime}$.

In $k$-ary $n$-cube networks, deadlock can be formed involving wraparound channels. This type of deadlock may not be detected by the rules above if it does not include sufficient number of turns. We take a simple approach for detecting such deadlock by regarding wraparound channel usage along the same dimension as a 180-degree turn, thus increasing count by two for COUNTING scheme. For BITSET scheme, it is treated as if the packet is making a 180-degree turn through one higher dimension, thus setting TBs corresponding to those two dimensions. The detailed description is omitted due to the space constraint.

\section{Performance}

This section presents simulation results of the proposed schemes and the scheme in [8] which is considered, to our knowledge, as most efficient in reducing the 


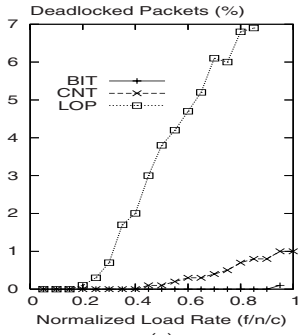

(a)

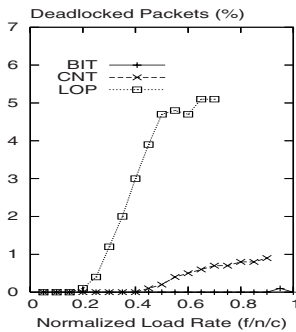

(b)

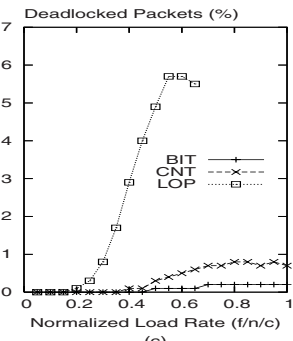

(c)

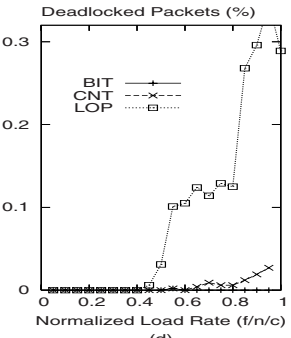

Fig. 1. Percentage of packets detected as deadlocked (a) $T O=16$ cycles, $16 \times 16$ meshes. (b) $T O=16$ cycles, $8 \times 8 \times 8$ meshes. (c) $T O=16$ cycles, $16 \times 16$ tori. (d) $T O=128$ cycles, $16 \times 16$ meshes.

number of false deadlock detections. The simulations are run on $16 \times 16$ and $8 \times 8 \times 8$ mesh and torus networks. Channels are with three virtual channels of buffer depth of two flits. The routing algorithm is minimal and fully adaptive. Packets are 32 flit-sized and their destinations are assumed uniformly distributed. We assume one clock cycle each for transmission of a flit over a channel, decoding a control flit, and crossing a switch. The statistics have been gathered after executing the program for 50000 clock cycles. The result of the first 10000 cycle is discarded for the initial transient period. Packets are generated exponentially with varying injection rate where the same rate is applied to all nodes. A packet presumed as deadlocked is ejected from the network and re-injected later when any of its requested channel resources is available.

We measured the percentage of packets detected as deadlocked by each strategy for varying normalized load rate of flits per node per cycle (f/n/c). Figure 1 shows the results for two TO thresholds of 16 and 128 clock cycles. The results of [8] are indicated with the legend 'LOP' and those of COUNTING and BITSET schemes with 'CNT' and 'BIT', respectively. $T O_{F}$ threshold for forwarding probes is set to two cycles for all experiments. The four figures show similar behaviors approximately. As expected, the percentage increases with the load rate. BIT detects almost no deadlock except for torus networks. Overall, CNT performs better than LOP, its percentage being approximately as much as eight times lower for meshes and eleven times lower for tori. It is shown that for large $T O$ such as 128 cycles, LOP yields less than 0.3 percentage of packets presumed as deadlocked, even at high loads for meshes. The results for CNT for the three network types are almost comparable, although packets would turn more often in 3D networks.

Normalized accepted traffic measured in flits per node per cycle is presented in Figure 2 for 2D meshes. All three schemes perform comparably in most cases except for high loads and TO of 16 cycles, at which the throughput of LOP drops drastically. This is because LOP detects too many packets as deadlocked for that network condition, as shown in Figure 1(a). Note that for other network conditions, the difference in the number of deadlocked packets has no significant effect on throughput. 


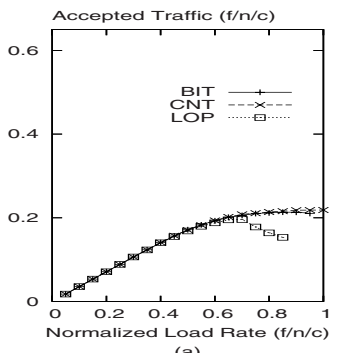

(a)

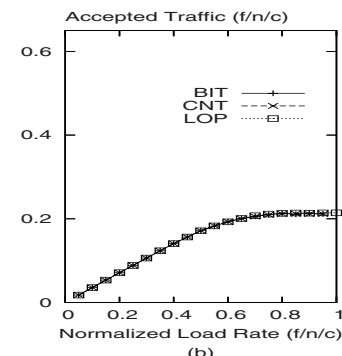

(b)

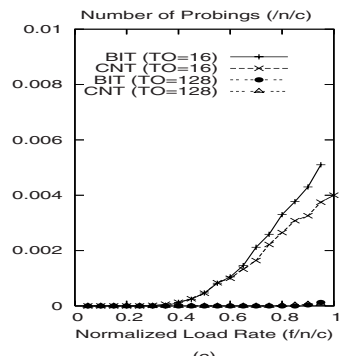

(c)

Fig. 2. 16x16 meshes (a) Normalized accepted traffic when $\mathrm{TO}=16$ cycles. (b) Normalized accepted traffic when $\mathrm{TO}=128$ cycles. (c) Mean number of probe initiations per node per clock cycle.

Table 1. Mean number of probe transmissions per probing for COUNTING scheme

\begin{tabular}{|c|c|c|c|c|c|c|c|c|c|}
\hline Network & \multicolumn{7}{|c|}{ Normalized Load Rate } \\
\cline { 2 - 9 } Configurations & 0.1 & 0.2 & 0.3 & 0.4 & 0.5 & 0.6 & 0.7 & 0.8 & 0.9 \\
\hline \hline 16x16 meshes and TO=16 cycles & $\mathrm{N} / \mathrm{A}$ & 5.6 & 6.6 & 6.2 & 6.3 & 6.4 & 6.6 & 6.6 & 6.6 \\
16x16 meshes and TO=128 cycles & $\mathrm{N} / \mathrm{A}$ & $\mathrm{N} / \mathrm{A}$ & $\mathrm{N} / \mathrm{A}$ & $\mathrm{N} / \mathrm{A}$ & 6.3 & 5.5 & 6.7 & 7.3 & 8.5 \\
\hline 8x8x8 meshes and TO=16 cycles & $\mathrm{N} / \mathrm{A}$ & 4.5 & 3.9 & 3.8 & 3.9 & 4.1 & 4.2 & 4.3 & 4.4 \\
8x8x8 meshes and $\mathrm{TO}=128$ cycles & $\mathrm{N} / \mathrm{A}$ & $\mathrm{N} / \mathrm{A}$ & $\mathrm{N} / \mathrm{A}$ & 2.8 & 3.4 & 4.0 & 4.3 & 4.4 & 4.5 \\
\hline
\end{tabular}

As the proposed schemes utilize a control packet, we measured its load on the router through the number of probings and the number of probe transmissions per probing. The number of probings initiated by a node per cycle is shown in Figure 2(c) for 2D meshes. Obviously, a node tends to initiate probings more often with higher loads but less frequently with higher thresholds. In particular, there is a significant difference between the results for the two thresholds. It is observed for $T O$ of 128 cycles that there is virtually no probing activity regardless of the network load. For $T O$ of 16 cycles, it is noted for both schemes that a node initiates approximately two probings per 1000 clock cycles at the saturated load and no more than five probings at extremely high loads. The reason for the slight difference between the results of the two schemes at high loads for $T O$ of 16 cycles is due to the fact that CNT detects more deadlocks than BIT as shown in Figure 1(a). That is, it facilitates blocked packets to proceed, since more packets are ejected from the highly loaded network. This reduces the need for probe initiations. BIT and CNT schemes showed similar results for the other network configurations.

Table 1 shows the number of probe transmissions for a probing. In general, more probes are transmitted for $2 \mathrm{D}$ meshes than for $3 \mathrm{D}$ meshes. This is simply because packets have more routing adaptivity and are less blocked in 3D meshes. It is noted that the number of probes tends to increase with the load rate for both networks, especially for TO of 128 cycles. For $T O$ of 16 cycles, blocked packets are dissipated promptly by more frequent probings than for 128-cycle $T O$, which leads to lower possibility of forwarding probes. 


\section{Conclusions}

This paper proposed enhanced mechanisms for deadlock detection in wormholerouted direct networks. Different from the previous schemes, the proposed schemes do not solely rely on the threshold value. A control packet propagates to find out the presence of deadlock. As the control packets traverse only along inactive channels, they virtually do not disturb normal packet progression. Simulation studies are conducted to compare the performance of the proposed schemes with that of the scheme which, to our knowledge, is most efficient in reducing the number of false deadlock detections. The simulation results demonstrate that the suggested schemes yield a substantial decrease in the number of deadlock detections in various network conditions. Consequently, our schemes outperform the previous scheme in terms of the network throughput irrespective of the time-out threshold.

\section{References}

1. Al-Tawil, K.M., Abd-El-Barr, M., Ashraf, F.: A survey and comparison of wormhole routing techniques in a mesh network. IEEE Network 11(2) (1997) 38-45

2. Park, H., Agrawal, D.P.: A generic design methodology for deadlock-free routing in multicomputer networks. Journal of Parallel and Distributed Computing 61(9) (2001) 1225-1248

3. Chiu, G.M.: The odd-even turn model for adaptive routing. IEEE Trans. Parallel and Distributed Systems 11(7) (2000)

4. Duato, J.: A general theory for deadlock-free adaptive routing using a mixed set of resources. IEEE Trans. Parallel and Distributed Systems 12(12) (2001) 1219-1235

5. Duato, J.: A necessary and sufficient condition for deadlock-free adaptive routing in wormhole networks. IEEE Trans. Parallel and Distributed Systems 6(10) (1995) $1055-1067$

6. Glass, C.J., Ni, L.M.: The turn model for adaptive routing. Journal of the ACM 41(5) (1994) 874-902

7. Kim, J., Liu, Z., Chien, A.: Compressionless routing: a framework for adaptive and fault-tolerant routing. IEEE Trans. Parallel and Distributed Systems 8(3) (1997) 229-244

8. Martinez, J.M., Lopez, P., Duato, J.: FC3D: flow control-based distributed deadlock detection mechanism for true fully adaptive routing in wormhole networks. IEEE Trans. Parallel and Distributed Systems 14(8) (2003) 765-779

9. Martinez, J.M., Lopez, P., Duato, J.: A cost-effective approach to deadlock handling in wormhole networks. IEEE Trans. Parallel and Distributed Systems 12(7) (2001) 716-729

10. Pinkston, T.M.: Flexible and efficient routing based on progressive deadlock recovery. IEEE Trans. Computers 48(7) (1999) 649-669

11. Pinkston, T.M., Warnakulasuriya, S.: Characterization of deadlocks in k-ary n-cube networks. IEEE Trans. Parallel and Distributed Systems 10(9) (1999) 904-921

12. Schwiebert, L., Jayasimha, D.N.: A necessary and sufficient condition for deadlockfree wormhole routing. Journal of Parallel and Distributed Computing 32 (1996) $103-117$ 